Sheena K. Aurora

Received: 20 November 2003

Accepted in revised form: 24 May 2004

S.K. Aurora (四)

Swedish Headache Center,

Swedish Neurosciences Institute,

1221 Madison, Suite 1026,

Seattle, WA 98116, USA

e-mail: sheena.aurora@swedish.org

Tel.: +1-206-215-2243

Fax: +1-206-215-2245

\title{
Chronic daily headache: recent insights from functional imaging
}

\begin{abstract}
New imaging technology allows us to study neurologic disorders that have had no previous structural basis. There have been recent reports on the involvement of nociceptive pathways in daily headache. A systematic review was performed using key words "chronic daily headache" and "imaging".

This paper reviews the literature on
\end{abstract}

imaging studies performed on daily headache with emphasis on the new imaging technology.

Key words Daily headache • Functional neuroimaging

\section{Introduction}

Headache on a daily or a near daily basis occurs in about $5 \%$ of the general population. Chronic daily headache $(\mathrm{CDH})$ is a descriptive term used for a heterogeneous group of conditions, which have headache on a daily or almost daily basis as a common feature. The term daily headache implies head pain on a daily or near daily basis and according to the International Headache Society (IHS), more than 15 days out of the month [1]. The new IHS to date has accepted a uniform classification, similar to the one that had been proposed by Silberstein et al. [2]. This new classification includes working criteria for chronic tension-type headache (TTH), new daily persistent headache (NDPH), hemicrania continua and chronic migraine. Of this classification, the latter, chronic migraine, is the most prevalent.

Hemicrania continua is a rare disorder which afflicts women more than men. The clinical features include unilateral headache, autonomic features similar to cluster headache and an exquisitely sensitive response to indomethacin [3]. The underlying pathogenetic mechanisms remain unclear. Similarly, the mechanisms of NDPH are unclear although patients are more likely to recall the time the headache started and antecedent illnesses such as viruses may produce persistent sensitisation of peripheral or central nociceptive pathways. This paper will concentrate on imaging of this chronic migraine, as this is the most prevalent subtype of chronic headache.

In most patients chronic migraine evolves from episodic migraine (EM). The underlying mechanisms of chronic migraine are not well known but medication overuse has been described implicitly with the popular term "transformed migraine" [4]. Routine imaging for typical chronic headache is usually normal with no evidence for structural abnormality in the presence of a normal neurological examination $[5,6]$. There has been an increasing body of evidence for involvement of nociceptive pathways in $\mathrm{CDH}$ and migraine. The first reports came from Raskin et al. who observed that a migraine-like 
headache developed in patients with electrode implantation in the periaqueductal grey (PAG) [7]. Recently the rostral brainstem has been identified as being specifically involved in migraine $[8,9]$. The influence of the PAG on the trigeminovascular nociception has also been studied using neurophysiological studies [10]. Using a cat model, Knight and Goadsby demonstrated a reduction in evoked trigeminal neuronal activity by stimulation of the PAG both to ipsilateral and contralateral stimulation and well localised to the ventrolateral PAG. We can now use imaging technology to identify both structural abnormalities in the pain pathways with particular attention to the PAG as well as identity differences in function of pain pathways in chronic headache.

\section{Case reports of structural abnormalities in nociceptive pathways in chronic headache}

Patient with multiple sclerosis

In a single case report a discrete sclerotic lesion in a patient with multiple sclerosis caused severe headache [11]. The patient was a 16-year-old who was previously headache free and then developed multiple sclerosis. A plaque was found in the region of the PAG which was thought to produce the migraine-like headache.

\section{Midbrain vascular malformation}

Recently a brainstem lesion in the area of PAG in a patient with $\mathrm{CDH}$ has been described [12]. The patient was found to have had a left-sided cavernoma in the dorsal midbrain region adjacent to the PAG.

Also, two members of a family with cavernous angiomas and mutations in chromosome $7 q$ had chronic migraine with angiomas in the upper brainstem as compared to other family members without chronic migraine who had supratentorial lesions [13].

From these case reports it is evident that patients with structural abnormalities in the PAG have more propensity towards developing a chronic headache disorder. We felt it was therefore worthwhile studying the nociceptive pathways of patients with daily headache using functional imaging. We had reported activation of the red nucleus (RN) and substantia nigra (SN) during a spontaneous migraine attack [14] (Fig. 1). The patient was studied during migraine with aura while he was experiencing a quadrantanopsia. Since then we have reported activation in these same structures, $\mathrm{RN}$ and $\mathrm{SN}$, in subjects with visual-

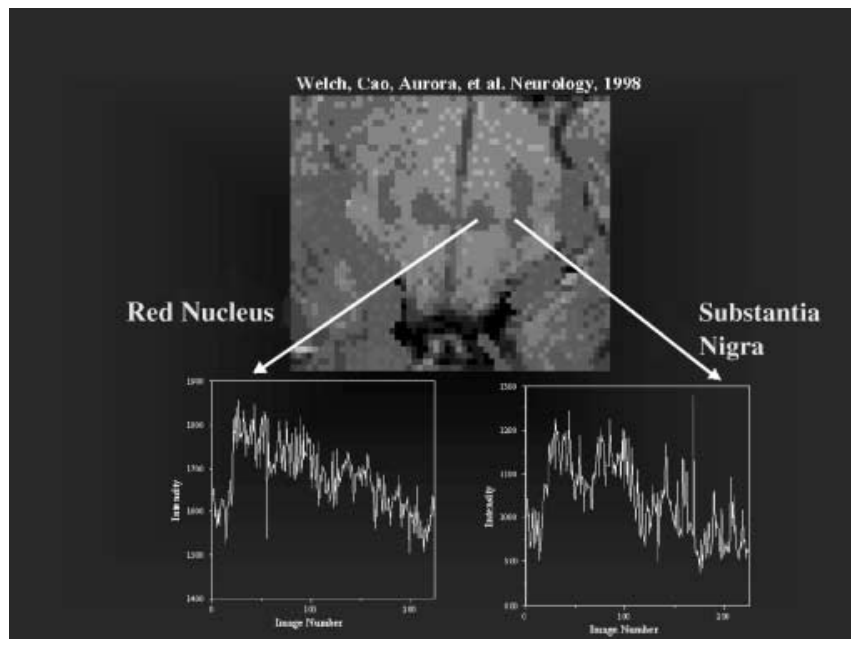

Fig. 1 Activation of red nucleus and substantia nigra in a patient with spontaneous migraine with aura

ly triggered migraine [15]. The RN and $\mathrm{SN}$ are best known for their functional roles in motor control. The RN however has also been associated with pain and or nociception [16]. Numerous animal studies have documented a response of RN neurons to a variety of sensory and noxious stimuli. In a PET study performed on normal volunteers during capsaicin-induced pain, ipsilateral activation of RN was documented. It remains to be clarified whether or not the $\mathrm{RN}$ or $\mathrm{SN}$ are involved in the pain pathways or in the motor response to pain.

These observations prompted study of iron homeostasis in the RN, SN and PAG of episodic and chronic migraine patients; elevation or decline in tissue iron is associated with altered cellular function [17]. We used high-resolution MR techniques to map the transverse relaxation rates R2 (1/T2), R2* (1/T2*) and R2' (R2*-R2) in brain, and in particular the PAG, RN and SN (Fig. 2). These measures are sensitive to shifts in the paramagnetic properties of free iron in brain tissue and blood [18]. Representative images containing the PAG, RN or SN of a subject are shown in Fig. 2. A positive correlation was noted between the duration of illness and the increase in R2' for the EM and CDH groups. The R2' are reflective of increased tissue iron levels in the PAG of EM with and without aura and CDH sufferers that further increase with duration of the disorder [19]. We discussed these findings as a possible cause of migraine and the burden of illness. Since this report there has been further evidence of iron metabolism changes in the RN; this study was performed in a large group of patients studied using 1.5 T MRI imaging [20]. This study further confirmed increase iron deposition in the RN and putamen in migraine patients.

Using similar technology outlined above we also had an opportunity to study a patient during CDH and EM. 


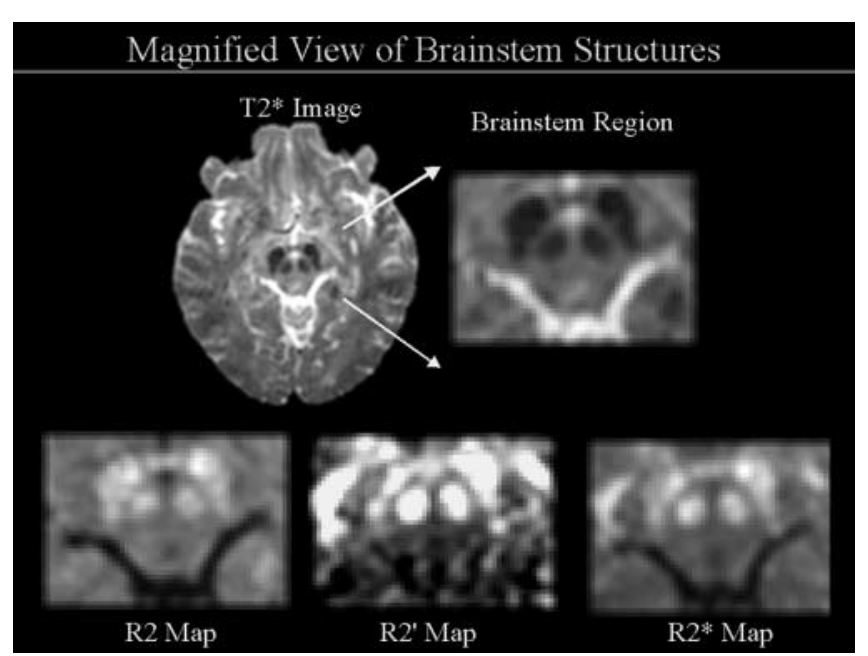

Fig. 2 Representative figure of measurement of iron homeostasis in brainstem structures by generation of R2*, R2' maps

This case accompanies this review. The patient had a history of episodic headaches, which met the International Headache Society (IHS) criteria for migraine without aura. During her $\mathrm{CDH}$ phase she was overusing ergotamine tartrate and extra-strength acetaminophen daily. The ergotamine tartrate and acetaminophen were withdrawn and the patient was treated with repeated dosages of intravenous dihydroergotamine for three days. On the second day the patient became headache free and subsequently reverted to EM. The patient was imaged in the interictal period during her $\mathrm{CDH}$ and EM phase. During CDH phase the patient's iron levels in the RN and $\mathrm{SN}$ were similar to those of other patients with $\mathrm{CDH}$ and then reverted to levels similar to EM. The PAG however had elevated R2' levels similar to those reported in $\mathrm{CDH}$ and other subjects with EM.

This case exemplified functional neuroimaging changes in nociceptive pain pathways during $\mathrm{CDH}$ and EM. The RN and SN both showed activation as measured by increased flow and hyperoxia during the $\mathrm{CDH}$ phase with normalisation during EM. We can therefore hypothesise that the analgesic overuse led to dynamic changes of activation in these structures. The PAG unfortunately could not be clearly delineated during $\mathrm{CDH}$ but showed an increased iron deposition during EM. This is in keeping with our previous finding that iron homeostasis is selectively, permanently and progressively impaired in the PAG indicative of a permanent dysfunction in EM.

From all the evidence thus far the PAG plays a pivotal role in both EM and $\mathrm{CDH}$. We have discussed first the structural abnormalities in this structure leading to a propensity for daily headache. This has further been strengthened by the changes in iron homeostasis described by the functional neuroimaging. The midbrain PAG is an anatomically heterogeneous, functionally diverse region of densely layered neurons surrounding the aqueduct of Sylvius [21]. Receiving input from the frontal cortex and hypothalamus, and projecting to the rostral ventromedial medulla thence to the medullary and spinal dorsal horn, the PAG is the centre of a powerful descending antinociceptive neuronal network. Further, the PAG can be considered a major nodal point in the central nervous system (CNS), regulating autonomic adjustments to antinociceptive, autonomic and behavioural responses to threat.

The dysfunction of PAG in migraine may explain why overuse of analgesics in EM is likely to result in $\mathrm{CDH}$. In animal experiments the PAG has been shown to be intimately involved with analgesia [22]. The PAG is also intimately involved in the behavioural response to opiate withdrawal [23]. Thus a dysfunctional PAG in patients who are inherently headache-prone may develop daily headaches when exposed to analgesics. This has been demonstrated in population-based studies and in a recent systematic study performed on a group of subjects using chronic analgesics for arthritis [24]. It could be therefore hypothesised that analgesics, because of selective action on specific brain sites i.e., PAG, produce a reaction in the form of $\mathrm{CDH}$ due to a dysfunctional $\mathrm{PAG}$ in migraine [25].

These imaging studies have helped us identify abnormal nociceptive pathways in patients who have $\mathrm{CDH}$, but further studies will be necessary to document the dynamic changes we have reported in the single case report in a larger subset of patients.

\section{References}

1. - (2004) The international classification of headache disorders, 2nd edn. Headache Classification Committee of the International Headache Society. Cephalalgia 24[Suppl 1]:1-160
2. Silberstein SD, Lipton R, Solomon S, Mathew N (1994) Classification of daily and near daily headaches: proposed revisions to the IHS classification. Headache 34:1-7
3. Sjaastad O, Spierings EL (1984)

Hemicrania continua: another headache absolutely responsive to indomethacin. Cephalalgia 4:65-70 
4. Mathew NT, Stubits E, Nigam M (1982) Transformation of migraine into daily headache: analysis of factors. Headache 22:66-68

5. Wang HZ, Simonson TM, Greco WR, Yuh WTC (2001) Brain MR imaging in the evaluation of chronic headache in patients without other neurologic symptoms. Acad Radiol 8:405-408

6. Weingarten S, Kleinman M, Elperin L, Larson EB (1992) The effectiveness of cerebral imaging in the diagnosis of chronic headache. Arch Intern Med 152:2457-2462

7. Raskin NH, Hosobuchi Y, Lamb S (1987) Headache may arise from perturbation of brain. Headache 27:416-420

8. Weiller C, May A, Limmroth V, Juptner M, Kaube H, Schayk RV, Coenen HH, Diener HC (1995) Brainstem activation in spontaneous human migraine attacks. Nat Med 1:658-660

9. Bahra A, Matharu MS, Buchel C, Frackowiak RSJ, Goadsby PJ (2001) Brainstem activation specific to migraine headache. Lancet 357:1016-1017

10. Knight YE, Goadsby PJ (2001) The periaqueductal grey matter modulates trigeminovascular input: a role in migraine. Neuroscience 106(4):793-800

11. Haas DC, Kent PF, Friedman DI (1993) Headache caused by a single lesion of multiple sclerosis in the periaqueductal gray area. Headache 33:452-455
12. Goadsby PJ (2002) Neurovascular headache and a midbrain vascular malformation: evidence for a role of the brainstem in chronic migraine. Cephalalgia 22(2):107-111

13. Mathew NT, Kailasam J, Meadors L (2002) Cavernous angiomas in the "brain stem generator" areas but not in other brain sites causing chronic migraine in subjects with familial cavernous angiomas. Headache 42(5):447-448

14. Welch KMA, Cao Y, Aurora SK, Wiggins G, Vikingstad EM (1998) MRI of the occipital cortex, red nucleus, and substantia nigra during visual aura of migraine. Neurology 51:1465-1469

15. Cao Y, Aurora SK, Vikingstad EM, Patel SC, Welch KMA (2002) Functional MRI of the red nucleus and occipital cortex during visual stimulation of subjects with migraine. Neurology 59:72-78

16. Iadarola MJ, Berman KF, Zeffiro TA, Byas-Smith MG, Gracely RH, Max MB, Bennett GJ (1998) Neural activation during acute capsaicin-evoked pain and allodynia assessed with PET. Brain 121(5):931-947

17. Morris CM, Candy JM, Omar S, Bloxham CA, Edwardson JA (1994) Transferrin receptors in the parkinsonian midbrain. Neuropathol Appl Neurobiol 20(5):468-472

18. Gelman N, Gorell JM, Barker PB, Savage RM, Spickler EM, Windham JP, Knight RA (1999) MR imaging of human brain at $3.0 \mathrm{~T}$ : preliminary report of transverse relaxation rates and relation to estimated iron content. Radiology 210:759-767
19. Welch KMA, Nagesh V, Aurora SK, Gelman N (2001) Periaqueductal gray matter dysfunction in migraine: cause or the burden of illness. Headache 41:629-637

20. Kruit MC, van Buchem MA, Overbosch J et al (2002) Iron deposits in migraine: red nucleus and putamen involved? A population based case-control MR imaging study, the cameraproject. Cephalalgia 22:571

21. Smith GS, Savery D, Marden C et al (1994) Distribution of messenger RNAs encoding enkephalin, substance $\mathrm{P}$, somatostatin, galanin, vasoactive intestinal polypeptide, neuropeptide Y, and calcitonin gene-related peptide in the midbrain periaqueductal gray in the rat. J Comp Neurol 350:23-40

22. Criswell HE (1976) Analgesia and hyperactivity following morphine microinjection into mouse brain. Pharmacol Biochem Behav 4:23-26

23. Christie MJ, Williams JT, Osborne PB, Bellchambers CE (1997) Where is the locus in opioid withdrawal? Trends Pharmacol Sci 18:134-140

24. Bahra A, Walsh M, Menon S, Goadsby PJ (2002) Does chronic daily headache arise de novo in association with regular analgesic use? Headache 42(5):427

25. Minami M, Onogi T, Toya T, Katao Y, Hosoi Y, Maekeawa K, Katsumata S, Yabuuchi K, Satoh M (1994) Molecular cloning and in situ hybridization histochemistry for rat $\mu$ opoid receptor. Neurosci Res 18(4):315-322 